ОСОБЕННОСТИ АЛГОРИТМОВ ФИЗИЧЕСКИ КОРРЕКТНОГО РЕНДЕРИНГА ДЛЯ ИНТЕРАКТИВНОЙ АРХИТЕКТУРНОЙ ВИЗУАЛИЗАЦИИ

\author{
А.В. Чистяков \\ Южно-Уральский государственный университет, г. Челябинск, Россия
}

\begin{abstract}
По результатам проведенных исследований раскрыты особенности создания фотореалистичных изображений. Выявлены основные задачи для формирования физически корректных визуализаций архитектурной среды. Рассмотрены понятие и уравнение рендеринга, а также аспекты теории микроповерхностей. Выполнен сравнительный анализ алгоритмов рендеринга с учетом различных свойств поверхности. Представлены примеры изображений архитектурной среды, полученные различными методами рендеринга. Выявлены преимущества и недостатки методов рендеринга. Предложен наиболее эффективный метод рендеринга для интерактивной архитектурной визуализации в реальном времени.

Ключевые слова: архитектурная среда, виртуальная реальность, интерактивная визуализация, теория микроповерхностей, физически корректный рендеринг, фотореалистичные изображения.
\end{abstract}

В ряду современных тенденций актуально применение систем виртуальной реальности в архитектурном проектировании. Статические изображения готовых проектных решений заменяются интерактивными анимированными сценами, которые позволяют более наглядно представить особенности проекта в различных условиях, приближенных к реальным. Архитектор имеет возможность изменять положение и направление взгляда в трехмерной сцене в реальном времени, изменять заданные свойства виртуальных объектов архитектурной среды [9]. Но для создания фотореалистичных физически корректных изображений, в том числе в режиме реального времени, современному архитектору необходимо понимать принципы формирования таких изображений и особенности различных методов рендеринга [2].

Обычно процесс формирования физически корректного изображения предполагает решение нескольких задач. Во-первых, это разработка и подготовка моделей сцены с потенциально сложными компонентами: оптимизация полигональной сетки трехмерных объектов, создание высококачественных фотореалистичных текстур и текстурных разверток, настройка физически корректного отображения материалов поверхностей и текстурных координат, постановка источников света и расположение виртуальных точек обзора [8]. Вовторых, это настройка программных алгоритмов создания изображения и решение уравнения рендеринга для каждой точки формируемого растрового изображения с учетом всех компонентов модели трехмерной сцены [1]. В-третьих, это настройка программных алгоритмов улучшения изображения с последующей обработкой изображения: коррекция цвета и экспозиции, линзовые эффекты, устранение зернистости [3]. Решение такого перечня задач реализует процесс создания физически корректного изображения достаточно продолжительным, особенно в части настройки программных алгоритмов создания изображения и решения уравнения рендеринга для каждой точки формируемого растрового изображения.

В математическом смысле, рендеринг - это попытка решения с разной степенью точности уравнения, которое определяет общее количество света, испущенного из заданной точки в заданном направлении, учитывая функцию входящего излучения и двунаправленную функцию распределения отражения. Причем уравнение рендеринга не учитывает все явления освещения, а только корпускулярные свойства света и является общей моделью освещения для компьютерных изображений [6].

Пусть $L$ - это количество излучения поверхности в определенное время $(t)$ заданной длины волны $(\lambda)$ по направлению вектора $(\omega)$ в точке падения луча $(x)$. Тогда количество излучения $\left(L_{o}\right)-$ это сумма излучённого света $\left(L_{e}\right)$ и отражённого света, где количество отражённого света представлено интегральным уравнением по полусфере всех приходящих излучений $\left(L_{i}\right)$ в точке поверхности с отражательной способностью $\left(f_{r}\right)$, поглощаемых под заданным углом $\left(-\omega^{\prime} \cdot n\right)$. В математическом описании уравнение может быть представлено следующим образом:

$$
\begin{aligned}
& L_{o}(\mathrm{x}, \omega, \lambda, \mathrm{t})=L_{e}(\mathrm{x}, \omega, \lambda, \mathrm{t})+ \\
& +\int_{\Omega} f_{r}\left(\mathrm{x}, \omega^{\prime}, \omega, \lambda, \mathrm{t}\right) L_{i}\left(\mathrm{x}, \omega^{\prime}, \lambda, \mathrm{t}\right)\left(-\omega^{\prime} \cdot \mathrm{n}\right) d \omega^{\prime} .
\end{aligned}
$$

Функция отражательной способности поверхности настолько важна, что получила в теории рендеринга собственное название - двунаправленная функция распределения отражений или «bidirectional reflectance distribution function» (BRDF), является основным инструментом при 


\section{Краткие сообщения}

моделировании шероховатых поверхностей с заданными свойствами, такими как: необходимые углы отражения, углы наклона микрограней шероховатых поверхностей и их светопоглощающая и светоотражающая способности. Предложенные аналитические модели этой функции, включая модель отражения Ламберта и Кука-Торренса, часто используются в компьютерной графике и учитывают физические аспекты микрогеометрии моделируемых поверхностей [7].

Поверхности различных материалов в реальном мире имеют различную степень шероховатости. Эти микронеровности значительно больше длины световой волны, и вносят существенный вклад в освещение. Невозможно описать шероховатую плоскость одним вектором нормали, нормаль обычно описывает некоторое усредненное значение макроповерхности. От неровностей поверхности, с которыми пересекаются лучи, зависят зеркальное и рассеянное отражения. Из-за рассеивания, которое происходит внутри объекта, эффект шероховатости рассеянного отражения менее заметен. В результате от грубости поверхности и от угла падения лучей сильно зависит направление исходящих лучей [10]. Угол падения равен углу отражения, вектор $h$ описывает именно нормаль микрограни, которая дает вклад в освещение. Кроме того, часть света физически не достигнет микрограней, которые могли бы отразить его. Микрограни, чьи нормали отражают строго между направлением света и областью видимости, будут отражать видимый свет. Но не все микрограни, где нормали микроповерхностей и повернутые нормали совпадают, будут отображаться, так как некоторые из них будут заблокированные тенью либо замаскированы.

Рассмотрим наиболее популярную математическую модель отраженного света КукаТорренса, которая представляет поверхность в виде множества микрограней, ориентированных в разные стороны, где $\mathrm{v}$ - направленный вектор от поверхности в точку наблюдения, $n$ - макро- нормаль поверхности, $l$ - направление от поверхности к источнику света, $F$ - коэффициенты отражения Френеля, $G$ - функция распределения самозатенения и самоперекрытия, $D$ - функция распределения отраженного света с учетом микрограней.

$$
f_{r}(\mathrm{v})=\frac{F\left(v, h, f_{o}\right) G(v, l, h) D(h, \alpha)}{4(n \cdot v)(n \cdot l)} .
$$

Распределение функции самозатенения и перекрытия использует метод Смита. Главная особенность этого метода заключается в том, что количество потерянного света от источника света до поверхности и от поверхности до точки наблюдения будет симметрично относительно макронормали поврехности.

$$
\mathrm{G}_{1}(\mathrm{v}, \mathrm{m})=\chi^{+}\left(\frac{\mathrm{v} \cdot \mathrm{m}}{\mathrm{v} \cdot \mathrm{n}}\right) \frac{2}{1+\sqrt{1+\alpha_{\mathrm{g}}^{2} \tan ^{2} \Theta_{\mathrm{v}}}}
$$

Все микрограни математически описываются коэффициентом шероховатости (roughness), который обычно лежит в диапазоне от «0» до «1». При значении «0» поверхность идеально гладкая, и микрограней нет. При значении «1» микрограни распределены так, что равновероятно отражают свет по полусфере. На практике при рендеринге трехмерных сфер с различными коэффициентами шероховатости получаем соответствующий результат (рис. 1).

Функция распределения отражающих микрограней позволяет установить какой процент микрограней для просчитываемого пикселя изображения имеют нормаль, совпадающую с вектором микрограней:

$$
D(m)=\frac{\alpha_{g}^{2} \chi^{+}(m \cdot n)}{\pi \cos ^{4} \cos ^{4} \Theta_{m}\left(\alpha_{g}^{2}+\tan ^{2} \Theta_{m}\right)^{2}} .
$$

При рендеринге трехмерных сфер с различными коэффициентами шероховатости и использованием распределения отражения получается следующий результат (рис. 2).

Достаточно точно описывают законы, по которым происходит отражение и преломление луча

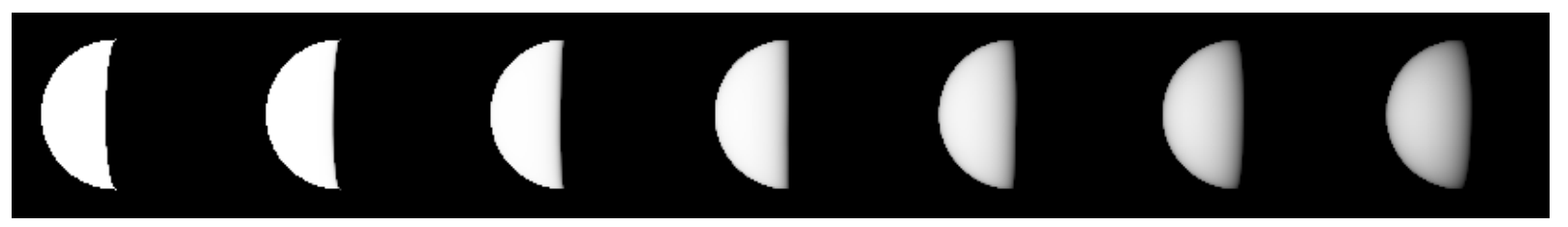

Рис. 1. Освещение трёхмерных сфер с различным коэффициентом шероховатости

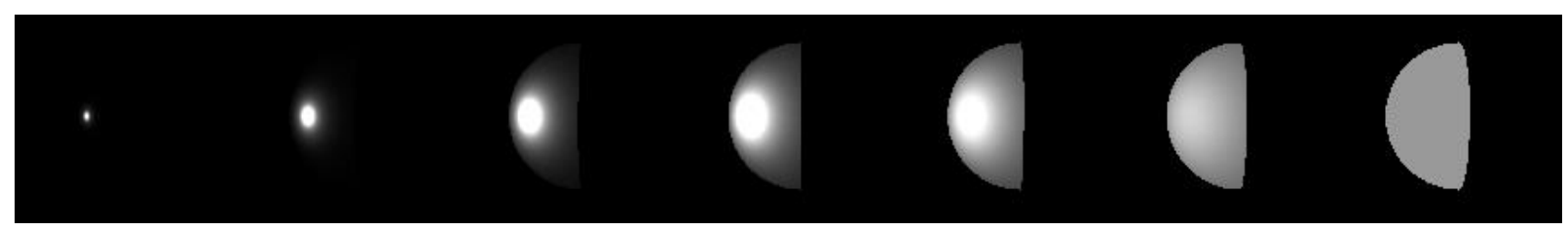

Рис. 2. Освещение трёхмерных сфер с различным коэффициентом шероховатости и использованием распределения отражения 
света, попавшего на границу раздела двух различных сред Формулы Френеля. Но данные формулы являются сложными для расчетов отражений в реальном времени, из-за чего для физически корректного рендеринга часто используют аппроксимацию Шлика.

$$
\begin{aligned}
& R(\Theta)=R_{0}+\left(1-R_{0}\right)(1-\cos \Theta)^{5} . \\
& R_{0}=\left(\frac{n_{1}-n_{2}}{n_{1}+n_{2}}\right)^{2} .
\end{aligned}
$$

В итоге, после учета всех составляющих двунаправленной функции распределения отражений получается следующий результат (рис. 3) [11].

Таким образом, при рассмотрении алгоритмов функции BRDF выявлено, как именно коэффициенты шероховатости поверхности влияют на отображение трёхмерных объектов при рендеринге, в том числе визуализацию архитектурной среды. В данном эксперименте не учитывалось глобальное освещение (GI). Это обобщенное название алгоритмов решения уравнения рендеринга, учитывающих как прямое излучение источников света, так и отраженное другими поверхностями в сцене (рис. 4). Достаточно просчитать диффузную карту глобальной освещенности один раз и использовать результаты просчета во всех последующих кадрах. Метод глобального освещения позволяет значительно сократить время получения нескольких изображений одной модели с различных видовых точек [12], например, модели интерьеров или архитектурной среды.

Рендеринг - это процесс, интенсивно исполь- зующий вычислительные ресурсы, который обычно используется для создания изображений или визуальных эффектов, в то время как рендеринг в реальном времени часто выполняется с использованием графических карт с аппаратными ускорителями [4]. Так и для систем виртуальной реальности необходимо выявить более эффективный метод рендеринга.

Освещение на основе изображений (Imagebased lighting), далее IBL, - это технология рендеринга, которая включает в себя захват всенаправленного представления информации о свете в реальном времени в качестве изображения с высоким динамическим диапазоном, обычно использующего специализированную камеру [14]. Полученное изображение проецируется на купол или сферу окружения трехмерной сцены [13] и используется для имитации освещения объектов в сцене (рис. 5).

Этот метод позволяет использовать очень подробное фотореалистичное изображение, для освещения сцены вместо того, чтобы пытаться точно моделировать освещение с использованием существующих методов рендеринга [5]. Преимущества метода IBL состоит в том, что не нужно решать интегральную часть по полусфере всех входящих излучений основного уравнения рендеринга, данные для освещения будут представлены текстурной картой окружения. Благодаря этому метод IBL в совокупности с другими методами рендеринга должны способствовать созданию реалистичных визуализаций и могут являться эффективным инструментом для интеграции объектов

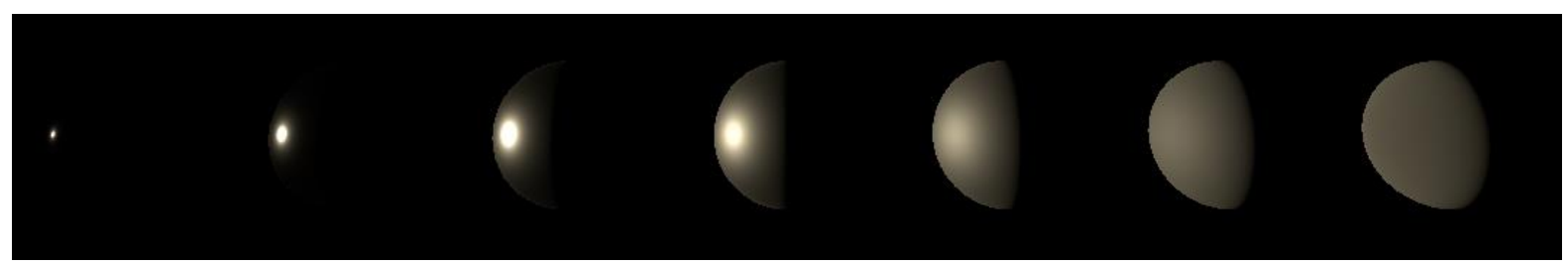

Рис. 3. Результат освещения трёхмерных сфер после учета всех составляющих двунаправленной функции распределения отражений

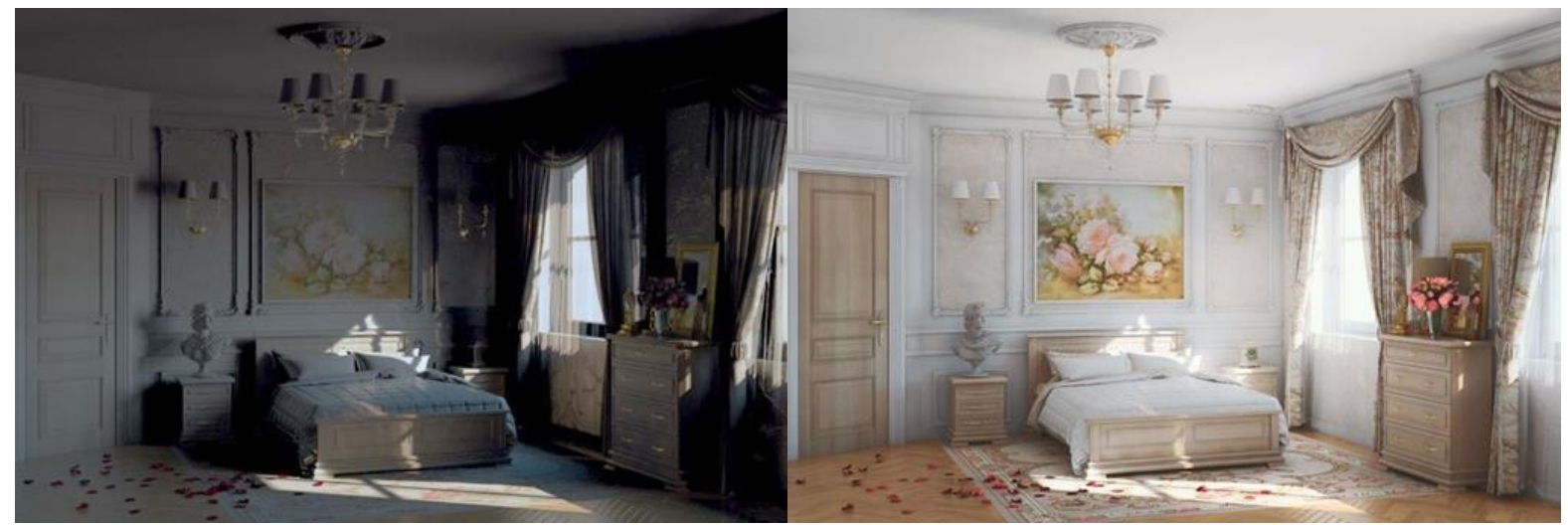

Рис. 4. Результат визуализации модели интерьера: BRDF слева, BRDF+GI справа 


\section{Краткие сообщения}

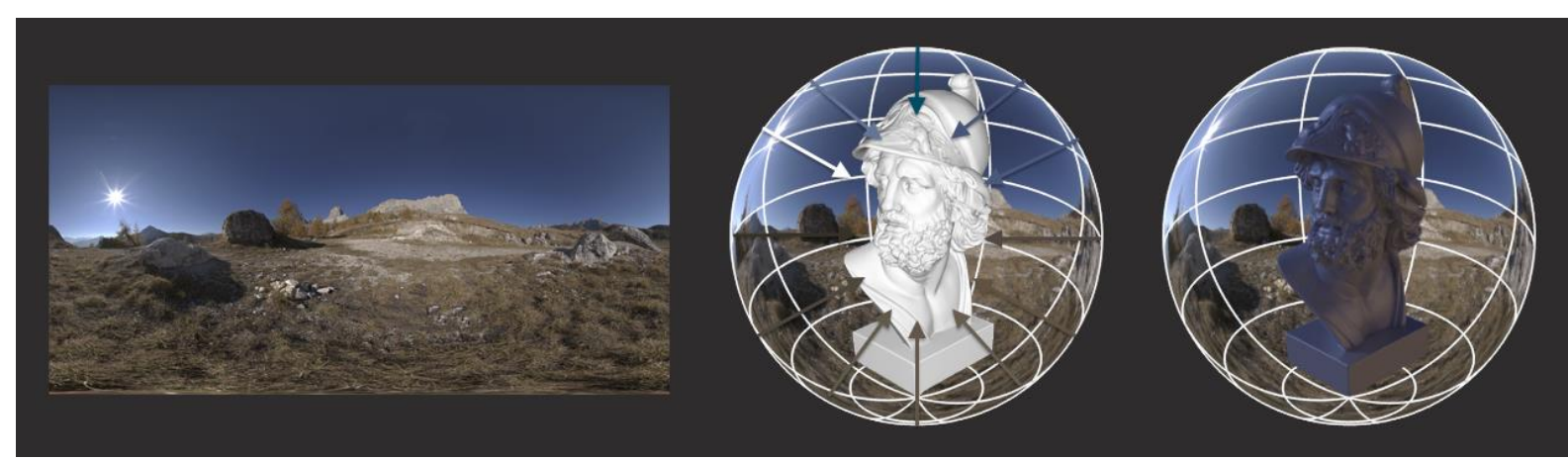

Рис. 5. Пример использования карты окружения для освещения трёхмерного объекта

компьютерной графики в системы виртуальной реальности, в том числе для физически корректной интерактивной визуализации архитектурной среды в реальном времени и реальном масштабе. Для более точных выводов необходимо провести дополнительный эксперимент.

В статье рассмотрены основные аспекты алгоритмов физически корректного рендеринга. Дальнейшая исследовательская работа заключается в анализе существующих программных решений для интерактивной визуализации архитектурной среды и создании собственного программного обеспечения для прототипирования архитектурной среды в реальном времени при помощи систем виртуальной реальности.

\section{Лumepamypa}

1. Дёмин, А.Ю. Основы компьютерной графики: учебное пособие / А.Ю. Дёмин. - Томск: Изд-во Томского политехнического университета, 2011.

2. Жданов, Д.Д. Объектно-ориентированная модель построения фотореалистичных изображений / Д.Д. Жданов. - http://www.graphicon.ru/ html/2013/papers/144-147.pdf

3. Калютов, А.В. Введение в фотореалистическую графику / А.В. Калютов. - СПб.: Политехника, 2015. - $118 \mathrm{c}$.

4. Мальцев, А.В. Методы и алгоритмы эффективного вычисления освещенности трехмерных виртуальных сцен в реальном режиме времени: дис. канд. физ.-мат. наук: 05.13.01 / А.В. Мальиев. - М., 2011 - С. 20-78.
5. Debevec, P. Image-Based Lighting / $P$. Debevec. - USC Institute for Creative Technologies, University of Southern California, 2016.

6. Immel, D.S. A radiosity method for nondiffuse environments / D.S. Immel; M.F. Cohen; D.P. Greenberg. - Siggraph, 1986.

7. Kajiya, J.T. The rendering equation / J.T. Kajiya. - Siggraph, 1986.

8. Kronander, J. Physically Based Rendering of Synthetic Objects in Real Environments /J. Kronander. Linköping University Electronic Press, Sweden, 2015.

9. LaValle, S.M. Virtual reality / S.M. LaValle. Illinois: Cambridge University Press, 2016.

10. McDermott, W. The Comprehensive PBR Guide. Vol. 1. Light and Matter: The theory of Physically-Based Rendering and Shading / W. McDermott. - Allegorithmic, 2015.

11. McDermott, W. The Comprehensive PBR Guide. Vol. 2. Light and Matter: Practical guidelines for creating PBR textures / W. McDermott. - Allegorithmic, 2015.

12. Ramamoorthi R. Precomputation-Based Rendering / R. Ramamoorthi. - Foundations and Trends in Computer Graphics and Vision, 2009.

13. Ren Z. Real-time Soft Shadows in Dynamic Scenes using Spherical Harmonic Exponentiation / Z. Ren, R. Wang, J. Snyder. - Microsoft Research, 2006.

14. Sloan, P. Precomputed Radiance Transfer for Real-Time Rendering in Dynamic, Low Frequency Lighting Environments / P. Sloan, J. Kautz, J. Snyder. - Computer Graphics (Proceedings of Siggraph), 2002.

Чистяков Андрей Викторович, магистр архитектуры, инженер, аспирант кафедры «Архитектура» Южно-Уральский государственный университет (Челябинск), perfidem@list.ru

Поступила в редакцию 26 апреля 2018 2. 


\title{
PECULIARITIES OF ALGORITHMS OF PHYSICALLY CORRECT RENDERING FOR INTERACTIVE ARCHITECTURE VISUALISATION
}

\author{
A.V. Chistyakov, perfidem@list.ru \\ South Ural State University, Chelyabinsk, Russian Federation
}

According to the results of the performed studies the peculiarities of creating photorealistic images were revealed. The main tasks were determined for forming of physically correct visualisations of architectural space. The concept and equation of rendering are considered, as well as the aspects of the theory of microsurfaces. A comparative analysis was performed for the algorithms of rendering with consideration to various surface properties. Examples of images of architectural space are given, obtained through various methods of rendering. The advantages and disadvantages of the methods of rendering are revealed. The most efficient method of rendering is suggested for interactive architectural visualization in real time.

Keywords: architectural space, virtual reality, interactive visualisation, theory of microsurfaces, physically correct rendering, photorealistic images.

\section{References}

1. Demin A.Yu. Osnovy komp'yuternoy grafiki: uchebnoye posobiye [Fundamentals of Computer Graphics: A Training Manual]. Tomsk, Tomskogo politekhnicheskogo universiteta Publ., 2011.

2. ZHdanov D. D. Ob"yektno-oriyentirovannaya model' postroyeniya fotorealistichnykh izobrazheniy. Object-Oriented Model of Building Photorealistic Images. Available at: http://www.graphicon.ru/html/ 2013/papers/144-147.pdf

3. Kalyutov A.V. Vvedeniye v fotorealisticheskuyu grafiku [Introduction to Photorealistic Graphics]. St. Petersburg, Politekhnika Publ., 2015. 118 p.

4. Mal'tsev A.V. Metody i algoritmy effektivnogo vychisleniya osveshchennosti trekhmernykh virtual'nykh stsen v real'nom rezhime vremeni. Diss... kand. fiziko-matemat. nauk. [Methods and Algorithms for Efficient Calculation of the Illumination of Three-Dimensional Virtual Scenes in Real Time Mode]. Moscow, 2011, 146 p.

5. Debevec P. Image-Based Lighting. USC Institute for Creative Technologies, University of Southern California, 2016.

6. Immel D.S., Cohen M.F., Greenberg D.P. A Radiosity Method for Non-Diffuse Environments. Siggraph, 1986.

7. Kajiya J.T. The Rendering Equation. Siggraph, 1986.

8. Kronander J. Physically Based Rendering of Synthetic Objects in Real Environments. Sweden, Linköping University Electronic Press, 2015.

9. LaValle S.M. Virtual reality. Illinois, Cambridge University Press, 2016.

10. McDermott W. The Comprehensive PBR Guide, vol. 1 Light and Matter: The theory of PhysicallyBased Rendering and Shading. Allegorithmic, 2015.

11. McDermott W. The Comprehensive PBR Guide, vol. 2 Light and Matter: Practical guidelines for creating PBR textures. Allegorithmic, 2015.

12. Ramamoorthi R. Precomputation-Based Rendering. Foundations and Trends in Computer Graphics and Vision, 2009.

13. Ren Z., Wang R., Snyder J. Real-time Soft Shadows in Dynamic Scenes using Spherical Harmonic Exponentiation. Microsoft Research, 2006.

14. Sloan P. Kautz J., Snyder J. Precomputed Radiance Transfer for Real-Time Rendering in Dynamic, Low Frequency Lighting Environments. Computer Graphics (Proceedings of Siggraph), 2002.

Received 26 April 2018

\section{ОБРАЗЕЦ ЦИТИРОВАНИЯ}

Чистяков, А.В. Особенности алгоритмов физически корректного рендеринга для интерактивной архитектурной визуализации / А.В. Чистяков // Вестник ЮУрГУ. Серия «Строительство и архитектура». - 2018. - Т. 18, № 3. - C. 73-77. DOI: 10.14529/build180311

\section{FOR CITATION}

Chistyakov A.V. Peculiarities of Algorithms of Physically Correct Rendering for Interactive Architecture Visualisation. Bulletin of the South Ural State University. Ser. Construction Engineering and Architecture. 2018, vol. 18, no. 3, pp. 73-77. (in Russ.). DOI: 10.14529/build180311 